\title{
Corela
}

Cognition, représentation, langage

13-1 | 2015

Vol. $13, \mathrm{n}^{\circ} 1$

\section{Les prédications en with : position et sémantisme}

\section{Gérard Deléchelle et Joëlle Popineau}

\section{OpenEdition}

\section{Journals}

Édition électronique

URL : http://journals.openedition.org/corela/3799

DOI : 10.4000/corela.3799

ISSN : 1638-573X

\section{Éditeur}

Cercle linguistique du Centre et de l'Ouest - CerLICO

\section{Référence électronique}

Gérard Deléchelle et Joëlle Popineau, « Les prédications en with : position et sémantisme », Corela [En ligne], 13-1 | 2015, mis en ligne le 30 juin 2015, consulté le 01 mai 2019. URL : http:// journals.openedition.org/corela/3799 ; DOI : 10.4000/corela.3799

Ce document a été généré automatiquement le 1 mai 2019.

\section{(c) (i) (2)(2)}

Corela - cognition, représentation, langage est mis à disposition selon les termes de la licence Creative Commons Attribution - Pas d'Utilisation Commerciale - Partage dans les Mêmes Conditions 4.0 International. 


\title{
Les prédications en with : position et sémantisme
}

\author{
Gérard Deléchelle et Joëlle Popineau
}

\section{Introduction}

1 Cette étude porte sur des énoncés dans lesquels on trouve des prédications en with, comportant des formes non-finies du verbe ou sans verbe de type with $\mathrm{SN}+\mathrm{SX}$, (SX représentant un élément prédicatif sur lequel on reviendra) comme :

(1) With the exams coming next, I have no time for a social life. [Quirk 1105]

(2) Collars are made of various materials, with leather being traditionally popular

for this purpose, although nylon collars are also now widely available. [BNC CJE 119]

(3) With so many children to support, they both have to work full time. [Quirk 1105]

(4) With the media all ears, he was very careful about what he said. [SZ Riehemann]

2 et elle se propose de préciser et d'interpréter le lien syntaxique et sémantique entre la proposition principale et la prédication en with, par exemple dans les deux schémas syntaxiques suivants :

\begin{tabular}{|l|l|}
\hline occurrences en anglais & schéma syntaxique \\
\hline (1) With the exams coming next, I have no time for a social life. & $\begin{array}{l}\text { with SN V-ing, } \\
\text { proposition p } \\
\text { prédication antéposée }\end{array}$ \\
\hline $\begin{array}{l}\text { (2) Collars are made of various materials, with leather being } \\
\text { traditionally popular for this purpose. }\end{array}$ & $\begin{array}{l}\text { Proposition p, with SN } \\
\text { V-ing } \\
\text { prédication postposée }\end{array}$ \\
\hline
\end{tabular}


Le point de départ de cette étude est la difficulté à proposer dans certains cas une traduction satisfaisante, comme l'indiquent Hélène et Jean Chuquet $^{1}$ (2006: 189), bien qu'on trouve en français des prédications introduites par avec ayant un sens voisin.

4 Les études précédentes ont porté essentiellement sur les prédications en with en position initiale, comme on le verra dans le tableau ci-dessous, tandis que les prédications postposées ont été moins étudiées. Notre but est de montrer les démarcations entre ces deux positions et l'influence de la position de cette prédication sur le sémantisme, ainsi que le rapport entre with et la notion de concomitance.

5 Nos exemples et occurrences de travail proviennent:

- du British National Corpus² (BNC) avec requête sélective,

- du Corpus of Contemporary American English (COCA),

- du Centre National de Ressources Textuelles et Lexicales (CNRTL),

- du corpus Collins Cobuild,

- du Oxford Advanced Learner's Dictionary (OALD),

- du Longman Dictionary of Contemporary English (LDOCE),

- et d'articles de journaux britanniques et américains actuels sur internet ${ }^{3}$.

\section{Diversité des prédications en with}

Dans Quirk et al., ces prédications en with ne sont mentionnées qu'en passant, par exemple en note d'une section consacrée aux « supplementive clauses » $(1985: 1124)$ ou avec certaines subordonnées (1985:1105, où figurent nos exemples (1) et (3)). McCawley (1983) y consacre plus de place. Elles sont parfois aussi mentionnées lors de débats sur les « free adjuncts » ou les constructions absolues, parfois dites « augmentées » parce qu'elles sont précédées d'une préposition, principalement with et parfois without. Les exemples cités jusqu'ici ne représentent pas l'ensemble des prédications en with étudiées par McCawley (1983) ou en avec, ces dernières ayant fait l'objet d'un long article de Ruwet (1978).

7 Divers types traités par Ruwet (1978) et McCawley (1983) sont mis en parallèle dans le tableau suivant par H. \& J. Chuquet $(2006: 192)$ :

\begin{tabular}{|c|c|c|}
\hline & Ruwet (1978) & McCawley (1983) \\
\hline $\begin{array}{l}\text { (a) } \\
\text { avec SN } \\
\text { part.prés } \\
\text { with NP V- } \\
\text { ing }\end{array}$ & $\begin{array}{l}\text { Avec son mari buvant comme un trou, } \\
\text { Bernadette est de plus en plus } \\
\text { malheureuse. }\end{array}$ & $\begin{array}{l}\text { With the postal rates going up next } \\
\text { week, you should answer these letters } \\
\text { right away. }\end{array}$ \\
\hline $\begin{array}{ll}\text { (b) } & \\
\text { avec } & \text { SN } \\
\text { SAdj } & \\
\text { with } & \text { NP } \\
\text { AdjP } & \end{array}$ & $\begin{array}{l}\text { Avec Paul malade, la réunion est remise } \\
\text { à plus tard. }\end{array}$ & $\begin{array}{l}\text { With Emil (obviously) afraid of snakes, } \\
\text { you shouldn't take him along on your } \\
\text { camping trip. }\end{array}$ \\
\hline
\end{tabular}




\begin{tabular}{|c|c|c|}
\hline $\begin{array}{l}\text { (c) } \\
\text { avec SN SP } \\
\text { with NP PP }\end{array}$ & $\begin{array}{l}\text { Avec Pierre pour guide, on va sûrement } \\
\text { s'égarer. } \\
\text { Avec la Castafiore en grande forme, il } \\
\text { va sûrement pleuvoir. } \\
\text { Avec Carter pour favori, ces élections } \\
\text { ne me concernent pas. }\end{array}$ & $\begin{array}{l}\text { With a girl in every port, Harry feels } \\
\text { pretty contented. } \\
\text { With the bus drivers on strike, we'll } \\
\text { have to ride our bicycles. } \\
\text { With Reagan as President, we're in grave } \\
\text { danger of war. }\end{array}$ \\
\hline $\begin{array}{l}\text { (d) } \\
\text { avec SN SN } \\
\text { with NP NP }\end{array}$ & $\begin{array}{l}\text { Avec mon cousin Jules Président de la } \\
\text { République, je vais pouvoir mener la } \\
\text { belle vie. }\end{array}$ & $\begin{array}{l}\text { With all your children students, you } \\
\text { must have a hard time making ends } \\
\text { meet. }\end{array}$ \\
\hline $\begin{array}{l}\text { (e) avec SN } \\
\text { with NP }\end{array}$ & $\begin{array}{l}\text { Avec une telle sécheresse, la récolte est } \\
\text { bien compromise. }\end{array}$ & $\begin{array}{l}\text { With this bad weather, we had better } \\
\text { stay home. }\end{array}$ \\
\hline $\begin{array}{l}\text { (f) } \\
\text { avec SN } \\
\text { Relative }\end{array}$ & $\begin{array}{l}\text { Avec mon livre qui ne se vend pas, je } \\
\text { n'arriverai pas à payer mes impôts. }\end{array}$ & \\
\hline $\begin{array}{l}\text { (g) } \\
\text { with NP } \\
\text { Adv }\end{array}$ & & $\begin{array}{l}\text { With John away, no one could get into } \\
\text { his apartment. }\end{array}$ \\
\hline
\end{tabular}

tableau auquel on aurait pu ajouter d'autres configurations :

\begin{tabular}{|c|c|c|}
\hline $\begin{array}{l}\text { (h) } \\
\text { Avec SN V } \\
\text { part. passé } \\
\text { With NP V } \\
\text { part. passé }\end{array}$ & $\begin{array}{l}\text { Avec leurs maisons complètement } \\
\text { détruites, ils ont tout perdu }\end{array}$ & $\begin{array}{l}\text { With their houses totally destroyed, they } \\
\text { have lost all their belongings }\end{array}$ \\
\hline $\begin{array}{l}\text { (i) } \\
\text { Avec SN à } \\
\text { Vinf } \\
\text { With NP } \\
\text { prep Vinf }\end{array}$ & $\begin{array}{l}\text { Avec mes examens à passer, je n'ai } \\
\text { plus de temps à perdre }\end{array}$ & $\begin{array}{l}\text { With children to look after, you can't do } \\
\text { what you like } \\
\text { With } 15 \text { minutes to go on the final day, it } \\
\text { looked as though it wouldn't be enough. }\end{array}$ \\
\hline
\end{tabular}

\section{Tests préliminaires et premier bilan}

9 Nous formulons l'hypothèse que la position respective des deux propositions joue un rôle dans l'interprétation sémantique pressentie.

10 Notre méthode de travail utilise des reformulations et traductions en anglais et en français. Celles-ci ont été choisies parce qu'elles montrent un lien syntaxique ou une interprétation suggérée ; elles ne sont pas des preuves pour autant. 
11 Les énoncés choisis subissent ainsi sept reformulations, à la fois sur l'énoncé de départ en anglais et sur la traduction française. Quelques exemples et leur comportement dans ces reformulations sont donnés ci-dessous ; ils mettent en œuvre reconstruction, inversion et traduction, de façon séparée ou combinée.

\subsection{Le premier test $(A)$ :}

Le premier test (A) reconstruit l'énoncé en anglais avec effacement du with; il est également appelé test d'annulabilité.

\begin{tabular}{|l|l|}
\hline $\begin{array}{l}\text { (1) With the exams coming next, I have no } \\
\text { time for a social life. }\end{array}$ & $\begin{array}{l}\text { (1A) ??The exams coming next, I have no time } \\
\text { for a social life. }\end{array}$ \\
\hline $\begin{array}{l}\text { (2) Collars are made of various materials, with } \\
\text { leather being traditionally popular for this purpose. }\end{array}$ & $\begin{array}{l}\text { (2A) Collars are made of various materials, leather } \\
\text { being traditionally popular for this purpose. }\end{array}$ \\
\hline $\begin{array}{l}\text { (3) With so many children to support, they both } \\
\text { have to work full time. }\end{array}$ & $\begin{array}{l}\text { (3A) [Having] so many children to support, they } \\
\text { both have to work full time. }\end{array}$ \\
\hline $\begin{array}{l}\text { (4) With the media all ears, he was very careful } \\
\text { about what he said. }\end{array}$ & $\begin{array}{l}\text { (4A) The media [being] all ears, he was very careful } \\
\text { about what he said. }\end{array}$ \\
\hline
\end{tabular}

13 On remarquera que si on veut supprimer with dans les énoncés (3) et (4), on est amené à introduire un support verbal attributif : have en $(3 \mathrm{~A})$ et be en $(4 \mathrm{~A})$, ce qui suggère déjà que sa présence peut ne pas être inutile. La suppression de with (ou de avec) n'est pas toujours possible dans certains des exemples figurant dans le tableau de chuquet (2006), par exemple en (e) :

(e) With this bad weather, we had better stay home.

(e') ?? This bad weather, we had better stay at home.

où une restructuration serait nécessaire pour rendre l'énoncé acceptable en introduisant soit une autre préposition comme because of, soit un auxiliaire :

(e") Because of this bad weather/the weather being so bad, we had better stay at home.

(e"')The weather being so bad, we had better stay at home.

Cependant, dans des énoncés du type de $(1 \mathrm{~A})$, l'omission de with n'est pas totalement exclue, en fonction du contexte, comme le montre l'exemple (a) de Mc Cawley dans le tableau ci-dessus: (With) the postal rates going up next week, you should answer these letters right away.

\subsection{Le deuxième test $(B)$}

Le deuxième test (B) propose de reconstruire les énoncés en anglais avec inversion des propositions.

(1) With the exams coming next, I have no time for a social life.
(1B) I have no time for a social life, with the exams coming next. 
(2) Collars are made of various materials, with leather being traditionally popular for this purpose.

(2B)? With leather being traditionally popular for this purpose, collars are made of various materials.

L'énoncé $2 \mathrm{~B}$ est sinon agrammatical, du moins sémantiquement mal formé.

On trouve cependant des prédications en with postposées supportant mieux l'antéposition avec cependant une fine nuance de sens entre (5) et (5B) :

(5) I can't do my homework with all this noise going on (http://livedic.com/lite/ search/with)

(5B) With all this noise going on, I can't do my homework.

Dans (5) la prédication postposée apporte une explication, alors que dans (5B), l'antéposition amène un sens causal, la principale étant la conséquence de la prédication antéposée.

\subsection{Le troisième test $(C)$}

Le troisième test $(\mathrm{C})$ consiste à reconstruire les énoncés en anglais avec un connecteur interprétant le sens implicité.

\begin{tabular}{|l|l|}
\hline $\begin{array}{l}\text { (1) With the exams coming next, I have no } \\
\text { time for a social life. }\end{array}$ & $\begin{array}{l}\text { (1C) Because/as the exams are coming next, I } \\
\text { have no time for a social life. }\end{array}$ \\
\hline $\begin{array}{l}\text { (2) Collars are made of various materials, with } \\
\text { leather being traditionally popular for this } \\
\text { purpose. }\end{array}$ & $\begin{array}{l}\text { (2C) Collars are made of various materials, among } \\
\text { which leather [is] traditionally popular for this } \\
\text { purpose. }\end{array}$ \\
\hline
\end{tabular}

21 Des connecteurs différents sont proposés suivant la position de la prédication. Pour les prédications antéposées, une interprétation causale ou plutôt de concomitance est suggérée, mais les connecteurs because ou as proposés pour reconstruire le sens pressenti explicitent un sens qui reste implicite avec with; il est à remarquer que ces deux connecteurs ne sont d'ailleurs pas eux-mêmes équivalents.

Dans le cas des prédications postposées comme (2C), among which explicite la relation ressentie, à savoir une notion sous-jacente de métonymie (partie/tout).

\subsection{Le quatrième test (D)}

Dans le quatrième test (D), with est traduit systématiquement par avec en français. Pour Chuquet (2006), with et avec ont de plus des cadres syntaxiques parallèles.

(1) With the exams coming next, I have no time for a social life.
(1D) ?Avec les examens approchant, je n'ai plus de temps pour les sorties ${ }^{4}$.

(1D') Avec l'approche des examens, je n'ai plus de temps pour les sorties.

(1D") Avec les examens [qui] approchent, 
(2) Collars are made of various materials, with leather being traditionally popular for this purpose.
(2D) ?Les colliers sont fabriqués à partir de différentes matières, avec le cuir étant généralement prisé pour cet usage.

(2D') ?Les colliers sont fabriqués à partir de différentes matières, avec le cuir généralement prisé pour cet usage

(2D") ?Les colliers sont fabriqués à partir de différentes matières, avec le cuir [qui est] généralement prisé pour cet usage donne des traductions correctes; on verra ci-après qu'il s'agit là de la meilleure traduction de ces prédications, quelle qu'en soit la position.

\subsection{Le sixième test $(F)$}

(1E) Les examens approchant, je n'ai plus de temps pour les sorties.

(2E) Les colliers sont fabriqués à partir de différentes matières, le cuir étant généralement prisé pour cet usage. 


\begin{tabular}{|l|l|}
\hline $\begin{array}{l}\text { (1) With the exams coming next, I have } \\
\text { no time for a social life. }\end{array}$ & $\begin{array}{l}\text { Les examens approchant, je n'ai plus de temps pour } \\
\text { les sorties. } \\
\text { (1F) Je n'ai plus de temps pour les sorties, les } \\
\text { examens approchant. }\end{array}$ \\
\hline $\begin{array}{l}\text { (2) Collars are made of various materials, } \\
\text { with leather being traditionally popular for } \\
\text { this purpose. }\end{array}$ & $\begin{array}{l}\text { Les colliers sont fabriqués à partir de différentes } \\
\text { uatières, le cuir étant généralement prisé pour cet } \\
\text { usage. ?Le cuir étant généralement prisé pour cet } \\
\text { usage, les colliers sont fabriqués à partir de } \\
\text { différentes matières. }\end{array}$ \\
\hline
\end{tabular}

30 Alors qu'en (1F) le test donne un énoncé correct (bien que moins naturel) pour les prédications antéposées, en (2F) l'inversion produit un énoncé sémantiquement inadapté, ce qui nous fait dire que la position de la prédication joue un rôle non négligeable dans les prédications postposées.

\subsection{Le septième et dernier test $(G)$}

31 Le septième et dernier test $(\mathrm{G})$ propose une reconstruction des énoncés en français avec un connecteur interprétant le sens pressenti.

\begin{tabular}{|l|l|}
\hline $\begin{array}{l}\text { (1) With the exams coming next, I have no } \\
\text { time for a social life. }\end{array}$ & $\begin{array}{l}(1 \mathrm{G}) \mathrm{Du} \text { fait de l'approche des examens, je n'ai plus } \\
\text { de temps pour les sorties. }\end{array}$ \\
\hline $\begin{array}{l}\text { (2) Collars are made of various materials, with } \\
\text { leather being traditionally popular for this } \\
\text { purpose. }\end{array}$ & $\begin{array}{l}(2 \mathrm{G}) \text { Les colliers sont fabriqués à partir de } \\
\text { différentes matières, dont le cuir, généralement } \\
\text { prisé pour cet usage. }\end{array}$ \\
\hline
\end{tabular}

Dans $(1 \mathrm{G}) d u$ fait que interprète un sens causal ressenti, non explicité dans with; (2G) est reconstruit en français avec dont, exprimant une relation métonymique, non explicitée dans with.

\subsection{Bilan des tests}

Certaines des reformulations ci-dessus ont été empruntées à Chuquet (2006), notamment la traduction systématique de with par avec en (1D/2D) ou l'effacement de with $(1 \mathrm{~A} / 2 \mathrm{~A})$ et de avec (1E/2E). Elles sont utilisées pour mettre en valeur certains éléments.

Les reformulations détaillées dans les sections 3.1. à 3.7. ne sont pas des traductions réelles, mais des efforts de reconstruction et de propositions de traductions. Il est bien entendu que la traduction est un témoin et non une preuve complète; si le traducteur explicite, ou s'il sous-traduit, il n'est pas complètement fidèle au texte. Tout comme les reformulations, la traduction est utilisée ici dans un but heuristique. 


\subsubsection{Pour les prédications en position initiale} formel entre les deux propositions, l'interprétation causale reste seulement suggérée et il en est de même lorsque les propositions sont inversées (1F). Enfin la traduction française par $d u$ fait de en $(1 \mathrm{G})$ explicitant un rapport causal implicite serait une alternative de traduction à (1E), mais les prédications en with antéposées n'impliquent pas toutes une lecture causale. La caractéristique des propositions participiales est qu'elles allient concision de forme et indétermination du sens.

\subsubsection{Pour les prédications en position finale.}

La traduction proposée (2E), où avec est élidé, illustre ce "décalage » entre les deux propositions qui se complète d'une valeur métonymique : des rapports sémantiques entre les substantifs des deux propositions (2F) se forment, renforcés par la valeur du with d'appartenance (2) qui est explicitée par among which, où among exprime « un ensemble dont fait partie quelque chose » ou une relation de métonymie. On verra cependant plus loin que les prédications en with postposées ne sont pas toutes du même type.

\section{Observations et remarques terminologiques}

Ce premier examen permet de rappeler plusieurs observations :

- on emploie ici le terme de "prédications (en with) » parce que la suite $\mathrm{SN}+\mathrm{SX}$ peut être considérée comme une prédication ou une relation prédicative d'une nature à définir, SX pouvant, on le voit dans le tableau ci-dessus, se réaliser comme SV (à forme non finie V-ing ou V-EN), SAdj, SP, SN, Adv. Le cas de prédications comme with this bad weather est intéressant parce que, bien que with soit suivi d'un simple SN, l'énoncé est à rapprocher des précédents, ce $\mathrm{SN}$ renvoyant non à une entité, mais à une situation ou un événement éventuellement qualifiés (cf. bad, ou telle) ;

- il s'agit de constructions détachées et mobiles, en général séparées de la principale p par une virgule (ou « comma intonation »).

- plusieurs travaux ont été consacrés (dans des cadres théoriques différents) à des énoncés comportant des configurations ne correspondant pas au schéma canonique des prédications à verbe fini : «free adjuncts », constructions absolues dans les grammaires traditionnelles, «supplementive clauses» pour Quirk et al. (1985), «small clauses» (en grammaire générative) ou encore prédications secondes. Les adjectifs «free» («free adjuncts»), «absolu» («constructions absolues»), «supplementive» renvoient au fait que ces constituants de la phrase complexe $\mathrm{q}, \mathrm{p}$ n'ont pas de lien syntaxique contraignant avec la principale $\mathrm{p}$ :

«When adverbial adjuncts are separated from the rest of the sentence by a clear break, they are called free adjuncts. The construction occurs more frequently in written than in spoken English » (Zandvoort $1977: 210$ ).

- d'un point de vue syntaxique, ces prédications sont situées, vis-à-vis de la principale $p$, en position « exophrastique » (ou extraprédicative), pour reprendre la terminologie utilisée par Guimier (1996) dans sa classification des adverbes (ou plus généralement des adverbiaux) : 
«Les adverbes exophrastiques sont des constituants externes à la phrase, en ce sens qu'ils ne participent pas à la construction de son sens référentiel, mais représentent des traces de l'intervention du locuteur qui commente tout ou partie de son énoncé ou de l'acte qui le produit» (Guimier $1996: 5-6)$.

$$
\begin{aligned}
& \text { totalement homogènes et qu'elles posent des problèmes de délimitation. Il n'est } \\
& \text { cependant pas inutile de s'interroger sur ce qui rapproche nos prédications en with } \\
& \text { d'exemples comme: }
\end{aligned}
$$

(6) Furious, the king ordered many arrests (Ferris 1993 : 37) 
circumstance has to be inferred ${ }^{9}$ from the context. » (Quirk et alii 1985, § 15.60 :

1124)

Bien que certaines de ces constructions soient proches des énoncés précédents, on notera que Quirk et al. n'évoquent qu'en note le cas des prédications en with que nous examinons ici, tandis qu'ils en donnent deux exemples (1 et 3 déjà cités) dans la section consacrée aux subordonnées dites « circumstantial» introduites par des subordonnants comme as, since, now that ou seeing that (p.1105):

(1) With the exams coming next, I have no time for a social life. (Quirk 1985 : 1105)

(3) With so many children to support, they both have to work full time (Quirk : 1105).

\section{Analyse des prédications en with SN V-ing}

\subsection{What's with with ? (McCawley 1983)}

Dans son article consacré aux constructions qu'il appelle "the with absolute » et dans lesquelles il qualifie parfois l'élément prédicatif de "nexus constituent» en faisant explicitement référence à Jespersen, McCawley recherche essentiellement la meilleure représentation de leur structure profonde. À partir de divers arguments (dont on trouvera le détail dans l'article), il propose une représentation du type de la figure 2 (qui pourrait aussi rendre compte de l'analyse de Jespersen) plutôt que celle de la figure 1 sous-jacente à la position de Ruwet (1978) :

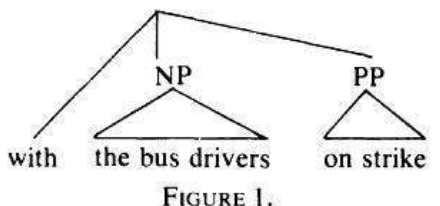

Figure 1.

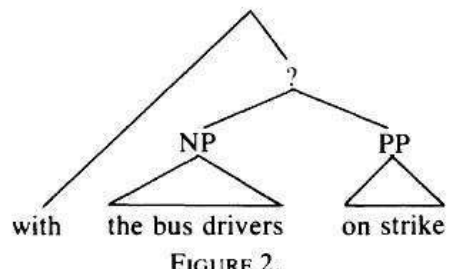

Figure 2.

(McCawley 1983 :272).

Il explique les différents cas de figure envisagés en posant soit un effacement en surface de be ou have soit une conversion de be en as dans « with Reagan as President». Il précise en outre qu'il s'en est tenu principalement aux énoncés où la prédication en with est antéposée, mais que ceci ne change pas l'analyse (même s'il n'exclut pas des différences de sens) :

«My impression is that absolute expressions in final position do not differ from those in initial position with regard to the issues discussed in this paper (which relate to the internal structure of the expression), though they do not play exactly the same roles in the sentences in which they occur. » (McCawley 1983: 271 - note 1).

et il renvoie à (Stump 1985) pour une étude du sémantisme de ces constructions.

\subsection{Participes $\mathrm{V}$-ing}

Avant de préciser le rôle de with, examinons celui de -ing dans les propositions participiales déjà abordé dans Deléchelle (2004a et b). Quirk et al. (1985 : 1121-1124) citent les prédications comportant un participe présent ou passé comme exemples de subordonnées adverbiales à valeur causale non introduites par un subordonnant : 
(7) John, knowing that his wife was expecting a baby, started to take a course on baby care.

(8) Persuaded by our optimism, he gladly contributed time and money to the scheme.

la participiale de (8) étant glosée par since he was persuaded, sans exclure en (7) une nuance temporelle. Un rapprochement est aussi fait en (7) avec les relatives non-restrictives (7b) ou les coordonnées (7c) :

(7b) John, who knew that his wife was expecting a baby, started to take a course on baby care

(7c) John knew that his wife was expecting a baby and he started to take a course on baby care.

51 Il y a une différence entre suggérer et exprimer explicitement un rapport causal. Cette valeur dérivée résulte moins des constructions syntaxiques que du sémantisme des propositions $\mathrm{p}$ et $\mathrm{q}$ et d'un processus interprétatif, inférentiel, comme le montrent les exemples suivants où l'on ne retrouve pas de valeur causale :

(9a) John opened the door, holding a book in his hand.

(9b) John, who was holding a book in his hand, opened the door.

(9c) John put the book on the table and opened the door.

52 Le participe (présent ou passé) étant la forme adjectivale du verbe, son interprétation se fera relativement au SN sujet et au temps de la principale. On attribue souvent à -ing une valeur de simultanéité ou de concomitance, mais il s'agit d'abord d'une marque de structuration $^{10}$. En outre, si on examine attentivement les exemples, on voit que les propositions participiales constituent plutôt un arrière-plan (temporel ou notionnel) au procès principal.

Nous ne reviendrons pas sur les différents facteurs jouant un rôle dans l'interprétation. Insistons plutôt sur le fait que, contrairement aux propositions introduites par un connecteur, la participiale évite de spécifier explicitement les relations sémantiques entre $\mathrm{p}$ et q:

«Selon le contenu lexical des deux propositions, V-ing pourra apporter à $\mathrm{p}$ une spécification situationnelle (temporelle), notionnelle (type explicatif), une caractérisation qualitative, parfois sur le mode additif (de type paratactique), sorte d'appendice parenthétique, d'afterthought. Cette dernière valeur, n'est bien sûr pas possible en antéposition, ce qui explique la difficulté à interpréter les participes postposés » (Deléchelle 2004a :134).

On oscille donc pour V-ing entre une valeur adjectivale et une valeur circonstancielle, de type concomitance, $\mathrm{V}$-ing pouvant selon les cas correspondre en français à un participe présent ou à un gérondif (en V-ant) ${ }^{11}$.

\section{3. « Constructions absolues » with SN V-ing (antéposition et postposition)}

On retrouvera des distinctions voisines dans les constructions absolues et les prédications en with SN V-ing, à cette différence près, et elle est d'importance, que la relation avec $\mathrm{p}$ sera moins étroite puisque le nexus aura cette fois un sujet exprimé en plus de l'élément prédicatif (verbe à une forme non-finie, groupe nominal, adjectival, prépositionnel ou un adverbe).

En (10) et (11) nous avons deux exemples de ce que la tradition appelle des constructions absolues non introduites par un connecteur (« unaugmented absolutes »): 
(10) It being Sunday, we decided to go for a walk.

(11) Dinner over, we decided to go for a drink.

En (12), la construction absolue (my sister a murderess) doit être introduite par with, avec la présence de as en (13) et d'une forme V-ing en (14) :

(12) How can I ever think of-of marriage? With my history. With my sister a murderess-or if not that, insane? [A. Christie]

(13) Then you must be married at Monte Carlo. Before the mayor. With myself as a witness.

(12) est intéressant parce que le premier with est une simple préposition alors que le second introduit une construction absolue, un with rendu nécessaire par le caractère attributif de la relation my sister-a murderess. With ne pourrait être omis que si on introduisait be: (my sister being a murderess).

(14) permet de faire le lien avec les participes en apposition, les deux constructions étant juxtaposées :

(14) She paused, toying with her cup, (with) her eyes watching the steaming black coffee.

Le rapprochement est ici facilité par le fait que dans la construction absolue le sujet her eyes, est en relation étroite avec le sujet principal she.

Comme on l'a vu, on trouve ces constructions aussi bien en antéposition qu'en postposition (cf. (1) et (2), bien que ces manipulations soient parfois soumises à des contraintes (cf. l'essai d'antéposition de with q en (2)) ou donnent lieu à des différences sémantiques plus ou moins marquées.

En antéposition, selon le co-texte ou le contexte, la prédication en with sert de cadre situationnel, temporel ou notionnel (logique) pour la validation de $\mathrm{p}$, ce qui explique qu'on lui attribue souvent une "valeur causale», comme semble le montrer la comparaison des deux exemples attestés suivants, issus d'une même publicité :

(15a) And with a bank of hundreds of questions on the CD-ROM version, each test will be significantly different from the last, so [ ...] (Publicité O.U.P.)

(15b) What's more, because the questions are selected from a database, each test is substantially different front the next. (ibid.)

Les deux énoncés ne sont cependant pas équivalents : si because marque explicitement un rapport causal, souligné ici par le choix de la conjonction et par sa position en tête d'énoncé, with se contente d'évoquer implicitement un tel rapport, qui relève cette fois de la concomitance notionnelle, la « contingence » de Quirk (1985), qu'on associe d'ailleurs plus à as qu'à because. C'est pourquoi nous avons dit à l'occasion du troisième test (C) que la glose en as ou because n'avait pas tout à fait le même sens que dans l'énoncé original (ces deux connecteurs n'étant d'ailleurs pas eux-mêmes équivalents, cf. Deléchelle 1989).

En (16) en revanche, l'emploi du participe passé et de once favorisent une lecture temporelle de la prédication en with:

(16) However, once in safety, with the door firmly shut behind her, her manner underwent a startling change. (M. Allingham, Black Plumes)

once jouant alors un rôle voisin de celui de with, l'un marquant l'accompli, l'autre la concomitance. Dans la publicité de (17) pour Time Magazine on pourrait voir aussi une nuance temporelle (quand vous achetez), conditionnelle (si vous achetez) ou composite (en achetant):

(17) With Time Magazine each week, you can expect to get (suit une liste des avantages que vous apportera (ou apporterait) cet achat...) 


$$
\text { eyes: }
$$

(19a) He walked up and down the dining room with his hands behind him and his head bent forward. (Körner 1956)

(19b) He walked up and down the dining room (with) his little eyes looking at me as if I was emerging from a deep sleep, dans les descriptions de personnages (parties du corps) ou de lieux, avec ou sans with. Dans ce type d'énoncés, la proposition réduite (avec ou sans with) a une valeur plus qualificative que circonstancielle ${ }^{12}$. En français, dans ce genre d'énoncés, on trouvera des constructions apposées comme: les yeux ouverts ou avec les yeux ouverts. Il n'est pas impossible de placer ces éléments en antéposition (comme en 20) en vue de mettre en valeur ces propriétés, tout en suggérant que ce sont elles qui rendaient l'endroit intemporel :

(20) Avec ses rues étroites, ses jardins tropicaux et ses maisons coloniales, l'endroit avait quelque chose d'intemporel. (G. Musso, 2006, Seras-tu là?: 179).

With its narrow streets, tropical gardens and colonial houses, the place had something timeless about $\mathrm{it}^{13}$.

Plus généralement, on trouvera en postposition des prédications en with qui s'inscrivent dans une relation métonymique ou méronymique, with venant ajouter un détail à $\mathrm{p}$ ou à un élément de $\mathrm{p}$ :

(21a) The city experienced its worst traffic congestion in years, with some drivers taking up to an hour just to move one mile forward. (Manhattan Gmat Forum).

(22a) There has been a shift from employment to unemployment since the beginning of the recession, with the young being the worst affected. [BNC]

(23a) of the prosecutions, 32 were successful, with one being quashed on appeal. [BNC]

72 C'est dans des cas comme ceux-ci ou comme (2) que l'antéposition ne donne pas un énoncé sémantiquement bien formé ou équivalent : 
(21b) ? With some drivers taking up to an hour just to move one mile forward, the city experienced its worst traffic congestion in years

(22b) ? With the young being the worst affected, there has been a shift from employment to unemployment since the beginning of the recession,

(23b) ? With one prosecution being quashed on appeal, 32 were successful. une heure est présenté comme un élément illustrant le contenu de $p$, un aspect de la situation décrite en $\mathrm{p}$. Si l'on voulait établir un rapport logique entre $\mathrm{p}$ et $\mathrm{q}$, q serait la conséquence plutôt que la cause de $\mathrm{p}$. Il suffit de revenir aux énoncés d'origine pour voir que la visée de discours est toute autre: with q en (22a) ajoute une information complémentaire, une spécification de p ou de l'un de ses éléments sur le mode mineur. On pourrait d'ailleurs remplacer la prédication en with par une coordonnée introduite par and marquant que $q$ vient après $p$, la présentation de l'information s'effectuant en deux temps. C'est encore plus net en (23a) où l'on évoque un élément d'un tout. La différence entre with $\mathrm{q}$ et and q est qu'avec with les éléments $\mathrm{p}$ et q ne sont pas sur le même plan, l'un étant sub-ordonné à l'autre.

Parfois with q introduit les conséquences, parmi d'autres, qui accompagnent ou sont associées à $\mathrm{p}$, rendant difficile l'antéposition de with $\mathrm{q}$. La concomitance n'implique pas forcément la consécution ${ }^{14}$.

(24a) The freeze continues with the prices of vegetables going up.

(24b) ? With the prices of vegetables going up, the freeze continues.

(24c) With the freeze continuing, the prices of vegetables are likely to go up.

Quand le lien métonymique entre les constituants devient moins étroit et moins évident, il ne restera que l'association entre les deux, with q ajoutant une information sur le mode additif, "continuatif », pour reprendre un terme associé aux relatives non-restrictives. On trouve fréquemment ce type d'exemples dans la presse qui éprouve le besoin de « compacter » l'information, sans pour autant tout mettre au même niveau :

(25) Welsh language and bilingual skills would be built into career programmes with parents being encouraged to take part (BNC).

(26) Temperatures will drop to $19 \mathrm{~F}(-4 \mathrm{C})$ in some areas of the country with fog blanketing large areas at the beginning of the week (Telegraph online Nov. 21, 2014)

(27a) Denham sat by the fire smoking, with Isaac dreaming at her feet. (Korner)

(27b) Denham sat by the fire smoking and (while) Isaac was dreaming at her feet.

(28) Second over the line was E. with S.K. third (compte-rendu d'une course)

En (28), on voit que la topicalisation de l'adjectif en début de phrase et inversement l'emploi de with $\mathrm{q}$ au lieu d'un simple and à la fin, permettent de hiérarchiser l'information, ou simplement d'ajouter une circonstance supplémentaire de type paratactique.

On voit donc que parmi tous les critères intervenant dans l'interprétation des prédications en with, l'ordre respectif des propositions joue un rôle non négligeable et en particulier quand with $q$ est placé après $p$.

\subsection{With et la concomitance ${ }^{15}$}

Il ressort de ces exemples que le rôle de with est de souligner non pas une concomitance temporelle, mais un repérage concomitant, un peu comme l'ablatif dit « absolu » en latin. Son emploi est souvent facultatif, mais il constitue un signal utile de subordination dans 
des exemples comme (30) où l'accumulation des circonstances concomitantes et des formes en -ing pourrait constituer un obstacle à la compréhension,

(29) Urbe deleta, hostis discessit (la ville détruite, l'ennemi s'éloigne)

(30) With Corky permanently on the premises, doing the Little Mother, and Gussie rolling up for practically every meal, and on top of that a gorilla like young Thos coming and parking himself in the spare room, you could scarcely expect him to bubble over with joie de vivre. These things take their toll. [P. G. Wodehouse]

Comment peut-on rapporter ces emplois de with à ses autres emplois? Comme pour les autres prépositions, les définitions des dictionnaires illustrent la prolifération des effets de sens (21 sens pour with dans le LDOCE et on pourrait faire la même observation à propos de avec) parfois assez éloignés les uns des autres. Ainsi, le Merriam Webster associe dans une rubrique moyen, cause, agent et instrument; dans une autre il signale une valeur temporelle et ailleurs il indique que with peut exprimer « an attendant fact or circumstance » (he stood with his hat on).

Même le regroupement de ces emplois sous quelques grandes rubriques comme comitatif, instrumental, manière, ne permet pas d'expliquer de façon homogène le fonctionnement véritable de la préposition, si on ne montre pas comment ces notions sont elles-mêmes construites.

81 Le débat sur les prépositions a souvent été centré sur une opposition entre des prépositions « incolores" (sémantiquement "vides » ou vidées, car grammaticales ou grammaticalisées) et d'autres au contenu sémantique plus marqué, with et avec se voyant attribuer une position intermédiaire. Auteur d'une thèse sur with, S. Gatelais rappelle que, historiquement, with est issu de deux étymons mid et wið, l'un statique qui correspondrait à among et l'autre dynamique comportant un mouvement de dissociation-association, wið ayant en vieil anglais une valeur adversative, qui n'a pas complètement disparu (cf. with all his faults, I still love him). Il souligne aussi que co-location n'est pas identification, les deux entités (le repère et le repéré) devant être référentiellement bien distinctes. Les deux prépositions ont un caractère méronymique: partie among tout, tout with/mid partie, sous-jacent à plusieurs emplois de with comme on l'a vu plus haut.

De nombreux exemples d'énoncés avec prédications en with finales reflètent ce sens méronymique :

(31) England is part of Europe, with France being their neighbours to the south and Norway being their Eastern neighbours (theguardian.com).

(31) est un exemple de lien patent, la France, la Norvège et l'Angleterre sont des pays membres (part) de l'Europe.

Dans (32) et (33), le lien partie/tout est inféré :

(32) The increase in gas was less substantial, with prices up as much as 1.3 cents a litre. [BNC]

(33) More than 30,000 people lost their job in November, the agency said, with the jobless rate accelerating from earlier in the year. [BNC]

Il faut reconstruire le raisonnement : le lien peut être redondant comme en (32) entre les prix qui augmentent et l'augmentation du prix du gaz, ou résultatif (il y a 30000 chômeurs de plus en novembre, le taux de chômage augmente par conséquent).

Dans (22) déjà cité, le préconstruit transparaît en filigrane, à la manière de la référence associative (Kleiber 1999):

(22) There has been a shift from employment to unemployment since the beginning

of the recession, with the young being the worst affected. 
Dans les catégories de chômeurs, on trouve plusieurs classes : les jeunes de moins de 25 ans, les 25-40 ans, et d'autres classes.

L'idée de «cause » associée à with dans les dictionnaires et les paraphrases de certains emplois peut s'expliquer à partir de paramètres de ce type, mais il ne s'agit que d'une valeur dérivée à laquelle with contribue à sa manière, conjointement avec d'autres facteurs.

Lemaréchal (1997) présente ainsi le problème à propos de avec:

En français «avec» qui marque des compléments de «manière ", " accompagnement » et " concomitance ", et non pas seulement d' "instrument ", n'a sans doute comme valeur propre [...] que l'expression d'une simple association. La valeur précise d' "instrument " relève d'inférences à partir d'un accord sémique, dans le contexte et la situation, entre l'action et le régime instrument potentiel de cette action». (1997: 116)

\subsection{With et as}

89 As est aussi associé dans certains emplois à la notion de " cause ", bien que, comme dans le cas de with, il ne s'agisse que d'une valeur dérivée, qui mérite elle aussi d'être examinée en relation avec la notion de concomitance, mais par un processus d'identification (as) plutôt que d'association-dissociation (with). C'est le cas pour certains de ses emplois comme conjonction, avec parfois une difficulté à séparer le temporel du causal, comme en (35) où c'est aussi parce qu'il continuait à neiger que la météo a jugé utile d'annoncer qu'elle prévoyait que des records pourraient être battus :

(34) As the snow continued to fall yesterday, meteorologists said there was a chance that the US 24-hour snowfall record of 76in could be broken (Telegraph, Nov 20, 2014).

Dans la phrase (35):

(35) As she grew older she gained in confidence [OALD]

donnée par le OALD comme exemple de co-temporalité ("while sth else is happening"), on ne peut exclure d'autres nuances sémantiques. Les énoncés en as (ou " comme " en français) peuvent prendre bien d'autres valeurs : temps (simultanéité, concomitance), manière, comparaison, similitude, analogie, ou diverses valeurs circonstancielles, sans qu'on puisse souvent les distinguer. L'énoncé exprime simplement une connexion entre les deux faits $P$ et $Q$. Le reste est une affaire d'interprétation, d'inférences reposant sur un savoir comme : « la confiance vient avec l'âge ", qui peut relever de données contextuelles ou pragmatiques. Ceci explique sans doute les problèmes soulignés par Le Querler (2000) et Vallée \& Khalifa (2000) pour traduire en français le as temporel.

Dans des emplois dits « prépositionnels » ou « prédicatifs » de as comme (36) et (37):

(36) Life as a student (titre d'un blog universitaire)

(37) 10 ways to have more money as a student, without working. (titre)

3 l'attribution d'une propriété (be a student) à un sujet (ici sous-entendu) peut aussi donner lieu en contexte à diverses interprétations : en tant qu'étudiant, quand on est étudiant. On comprend pourquoi Le Goffic (1991: 28) a pu qualifier comme de "marqueur de circonstance vague [qui] solidarise, cheville [...] les deux événements qu'il circonstancie ».

Si on peut parler de concomitance aussi bien pour as que pour with, comment peut-on les différencier? On a souvent observé qu'une des paraphrases possibles de with était have, alors qu'on associe généralement as à be, Emonds (1984:264) parlant de «copule 
prépositionnelle » («prepositional copula $»)^{16}$ pour des emplois comme (36). With opère une association-dissociation, as une identification plus ou moins étroite. Il n'en reste pas moins qu'on les retrouve assez souvent dans des contextes voisins, notamment dans des titres ou articles de presse :

(38) Winter to arrive with below-freezing icy blast (titre 1)

Temperatures will drop to $19 \mathrm{~F}(-4 \mathrm{C})$ in some areas of the country next week as winter arrives. (titre 2)

The first icy blast of winter will hit Britain next week as temperatures drop below freezing and Arctic winds being an end to the mild Autumn.

Temperatures will drop to $19 \mathrm{~F}(-4 \mathrm{C})$ in some areas of the country with fog blanketing large areas at the beginning of the week. (suite de l'article) (The Telegraph, Nov. 21, 2014)

\section{Bilan}

Comme on l'a signalé ailleurs (Deléchelle 2004a : 140), dans ces prédications la notion de concomitance peut intervenir à plusieurs niveaux :

- événementiel (simultanéité) ;

- syntaxique : prédications secondes ou détachées portant sur un terme ou sur l'ensemble de $\mathrm{p}$, avec un fonctionnement intermédiaire entre l'attributif et le circonstanciel mais n'exprimant pas une circonstance particulière ;

- discursif : prédications fournissant un repérage complémentaire éclairant l'énonciation principale, soit comme point de départ (en antéposition) soit comme prolongement qualificatif ou explicatif dans une relation souvent métonymique (en postposition).

Elle n'est donc pas synonyme de la simple simultanéité. Il s'agit plutôt d'une relation sémantique non-spécifiée, évoquant des caractéristiques ou une qualification d'un terme ou d'une prédication, sur le mode associatif.

Les valeurs sémantiques souvent associées à ces énoncés, comme la cause, résultent en fait d'inférences reposant sur de nombreux éléments d'ordre sémantique, syntaxique, prosodique ou discursif ${ }^{17}$. Le lien est volontairement sous-spécifié pour des raisons tenant à la visée discursive de l'énonciateur.

Parmi les facteurs évoqués par divers auteurs pouvant intervenir dans l'interprétation de ces énoncés, une place particulière a ici été accordée à la position des prédications en with, parce qu'il s'agit d'un problème peu étudié, mais non négligeable.

\section{BIBLIOGRAPHIE}

Adamczewski, H. \& Delmas C. 1982. Grammaire linguistique de l'anglais. Paris : A. Colin.

Cadiot, P. \& Furukawa N. (dir.) 2000. « La Prédication seconde », Langages 127.

Cadiot, P. 1997. « Avec ou le déploiement de l'éventail ». In Guimier G. (dir.). Texte et Co-texte.

Caen : Presses Universitaires de Caen : 135-155. 
Choi-Jonin, I. 2005. « La construction en avec en position détachée », Les Constructions détachées. Timisoara.

Chuquet, H. \& J. 2006. « De la circonstance à la cause: les prédications en with et leur traduction en français ». In Chuquet H. \& Paillard M. (dir.). Causalité et contrastivité, P.U. Rennes : 189-210.

Combettes, B. 1998. Les Constructions détachées en français. Paris : Ophrys.

Condamines, A. 2006. " Avec et l'expression de la méronymie : l'importance du genre textuel ». In Kleiber, G. Schnedecker, C. et Thyssen, A. (dir.). La relation "Partie - Tout". Peeters : 633-650.

Culioli, A. 1990. Pour une linguistique de l'énonciation 1. Paris : Ophrys.

Deléchelle, G. 1989. L'expression de la cause en anglais contemporain, étude de quelques connecteurs et opérations, thèse Paris III.

Deléchelle, G. 2004a. «Causalité et phrase complexe : prédications et circonstances concomitantes», Cercles 9, www. cercles.com : 121-142

Deléchelle, G. 2004b. « Ing Revisited ». In Hommage à H. Adamczewski. Editions E.M.A. : 20-32

Emonds, J. E. 1984. A Unified Theory of Syntactic Categories. Dordrecht-Cinnaminson: Foris Publications.

Ferris, C. 1993. The Meaning of Syntax: A Study in the Adjectives of English. London, New York: Longman.

Franckel, J. J. \& Paillard, D. 1999. « Considérations sur l'antéposition des syntagmes prépositionnels ». In Guimier C. (dir.) La thématisation dans les langues. Bern, Peter Lang : 277-295.

Gatelais, S. 2010. "With est-elle une préposition spatiale ?". In Delmas C. (dir.) Espace, temps en anglais, Faits de Langues. Paris : Ophrys :109-121.

Geis, M. \& Zwicky, A. 1971. « On Invited Inferences », Linguistic Inquiry 2 : 561-566.

Groussier, M.-L. 2001. « Pourquoi la préposition vieille-anglaise mid a-t-elle disparu au profit de with? Arguments en faveur d'une origine cognitive de cette disparition », Graat 24 : 21-37.

Guimier, C. 1996. Les adverbes du français : le cas des adverbes en -ment. Paris : Ophrys

Hamelin, L. 2013. « Dealing with with», Syntaxe et sémantique 1/2013 ( $\left.{ }^{\circ} 14\right)$. Presses

Universitaires de Caen : 67-83.

Herslund, M. 2000. « Le participe présent comme co-verbe », Langue française $127: 86-94$

Jespersen, O. 1933. Essentials of English Grammar. London : Allen and Unwin.

Khalifa, J. C. \& Vallée, M. 2000. « As temporel : opération d'identification ? Étude du marqueur et de ses traductions en français ». In Chuquet, J. (dir.) Complexité syntaxique et sémantique. Études de corpus, Les Cahiers FoReLL 14. Poitiers : 23-46

Kleiber, G. 1999. « Anaphore associative et relation partie-tout : condition d'aliénation et principe de congruence ontologique ». Langue Française 122 : 70-100.

Körner, R. 1956. « Strödda Bidrag till Comparative Grammatik IX », Moderna Sprak 50 : 449-463.

Kortmann, B. 1991. Free Adjuncts and Absolutes in English: Problems of Control and Interpretation. London/New York: Routledge.

Langages 1993, n 110, La couleur des prépositions, Larousse. 
Le Goffic, P. 1991. «Comme, adverbe connecteur intégratif : éléments pour une description ", Travaux Linguistiques du Cerlico, $\mathrm{n}^{\circ} 4$ (L'Adverbe dans tous ses états), Rennes : Presses Universitaires de Rennes $2: 11-31$.

Le Goffic, P 1997. « Formes en -ant et contexte ». In Guimier C., Co-texte et calcul du sens, Presses Universitaires de Caen : 123-133.

Le Querler, N. 1993. «Les Circonstants et la position initiale ». In Guimier C. (dir.). 1001 circonstants , P.U. Caen : 159-184.

Le Querler, N. 2000. «Paraphrase et traduction : L'exemple de when, as, quand et lorsque ».

Tunis. Publications de l'ENS : 215-230.

Lemaréchal, A. 1997. « Séries verbales et prépositions : Incorporation et décumul des relations», Faits de langues 9 : 109-118.

Mala, M. 2005. « Absolutes », Discourse and Interaction 1. Brno Seminar on Linguistic Studies in English: Proceedings.

McCawley, J.D. 1983. « What's with with », Language 59, 2: 271-287.

Miller, P. 1998. «Compléments et circonstants : Une distinction syntaxique ou sémantique ?», Cycnos 15 : 91-103.

Quirk, R. et alii. 1985. A Comprehensive Grammar of the English language. Londres : Longman. Ruwet, N. 1978. « Une construction absolue en français », Linguisticae Investigationes 11 : 165-210. Santin-Guettier, A.-M. 2005. «WITH : opérateur ambivalent? », Anglophonia, Université Toulouse Le Mirail : 173-184.

Seiler, H. J. 1974. «The Principle of Concomitance: Instrumental, Comitative and Collective», Foundations of Language 12 : 215-247.

Stump, G. T. 1985. The Semantic Variability of Absolute Constructions. Dordrecht : D. Reidel.

Zandvoort, R. W. 1977. A Handbook of English Grammar. $7^{\mathrm{e}}$ édition, Londres : Longman.

SITOGRAPHIE

thetelegraph.com

huffingtonpost.com

bbc.co.uk

theguardian.com

http://livedic.com/lite/search/with

\section{NOTES}

1. Nous adoptons comme eux l'expression « prédications en with ».

Les auteurs remercient les deux relecteurs anonymes pour leurs remarques et suggestions qui ont permis d'améliorer cet article et restent entièrement responsables de toute erreur qui subsisterait.

2. BNC et COCA sont des corpus en ligne.

3. Une sitographie est donnée dans les références.

4. Autre possibilité de traduction : Je n'ai plus de temps à consacrer aux sorties 
5. Bien que Ruwet (1978) donne dans le tableau déjà cité un exemple du type : avec SN V-ant X, des énoncés comme 1D sont difficilement acceptables comme traduction de with SN V-ing. On notera en revanche l'existence en français d'une (pseudo) relative (1D") qu'on ne trouve pas en anglais.

6. On trouve des exemples de phrases traduites contenant "avec l'approche de" (Dictionnaire anglais-français et moteur de recherche de traductions). Autre exemple: « Avec l'arrivée des premières chaleurs, c'est aussi les examens qui approchent " (site de l'Université de Genève).

7. Voir par exemple les numéros 31 et 32 de la revue Faits de langues (2008) sur la prédication.

8. Voir Langue française $\mathrm{N}^{\circ} 125$ (2000) sur l'apposition et 127 (2000) sur la prédication seconde

9. Cf. les « invited inferences » (« inférences suggérées ») de Geis et Zwicky (1971)

10. Cf. Goffic (1997:129) : «L'opposition entre un participe présent et une forme temporelle est plutôt du type « mineur » / " majeur » [...] il apparaît bien plus comme une forme d'économie, de relais, beaucoup plus dépendante des types de procès. ». Pour Adamczewski (1982), ing est un marqueur de subordination. Mc Cawley (1983) fait la même analyse pour le -ing des prédications en with (McCawley 1983 : 276).

11. Voir dans Herslund (2000) les valeurs différentes que peut prendre le participe présent français selon qu'il est antéposé ou postposé à p.

12. On sait en outre les difficultés que soulève la détermination du statut des compléments dits « de manière » (cf. Deléchelle 1989 : 751).

13. Les traductions proposées sont celles des auteurs.

14. Pour Culioli (1990: 200), la causalité associe consécution et concomitance.

15. Rappelons que c'est à propos de la préposition mit (with en allemand) que Seiler (1974) avait renouvelé le débat sur la notion de concomitance.

16. Si on s'interroge sur le statut de with dans ces prédications (préposition, conjonction ou préposition conjonctive ?), il est également difficile de préciser dans certains cas le statut de as. Les catégories grammaticales traditionnelles montrent ici leurs limites. L'opération importe plus que la catégorie.

17. Depuis quelques années des avocats font appel à des linguistes pour savoir comment interpréter (à leur avantage) le lien entre les deux propositions du deuxième amendement de la constitution américaine : "A well regulated Militia, being necessary to the security of a free State, the right of the people to keep and bear arms, shall not be infringed."

\section{RÉSUMÉS}

Cet article porte sur les " prédications en with ", énoncés prédicatifs à verbe non fini introduits par with, qui peuvent apparaître en antéposition (With the exams coming next, I have no time for a social life) ou en postposition (England is part of Europe, with France being their neighbours to the south). Leur position dans la phrase influe sur l'interprétation sémantique. En position initiale, le lien pressenti est un lien de concomitance, alors qu'en positon finale, la situation est plus contrastée : il peut s'agir d'un élément supplémentaire ou secondaire de la description ou d'une relation métonymique tissée entre des éléments des propositions. Des tests et reformulations permettent d'illustrer et décrire les caractéristiques de ces prédications peu étudiées, pouvant poser des problèmes de traduction. 
This paper deals with "with predications" i.e., clauses introduced by with followed by a nonfinite verb in the present participle, placed either before (e.g. With the exams coming next, I have no time for a social life) or after the main clause (e.g. England is part of Europe, with France being their neighbours to the south). The paper shows that depending on their position, the implied semantic link differs and the clause may suggest an accompanying circumstance to the main clause or a metonymic link between the contents of the two clauses

INDEX

Mots-clés : prédications en with, relations interpropositionnelles, position, interprétation sémantique, concomitance, métonymie

Keywords : with non-finite clauses, inter-clausal relations, semantic interpretation, accompanying clauses, metonymic link.

\section{AUTEURS}

\section{GÉRARD DELÉCHELLE}

LLL UMR 7270, Université de Tours

\section{JOËLLE POPINEAU}

DEA EA 2338, Université de Lorraine/LLL UMR 7270, Université de Tours 